\title{
Valva mitral heteróloga sem suporte: resultados clínicos a médio prazo
}

\author{
Mário O. VRANDECIC*, Bayard GONTIJO FILHO*, Fernando Antônio FANTINI*, Idail Costa MAR- \\ TINS Jr.*, Marcelo H. OLIVEIRA*, Sandra O. S. AVELAR*, Ozanam OLIVEIRA*, Erika \\ VRANDECIC*, Ektor VRANDECIC*, João Alfredo PAULA E SILVA*
}

Vrandecic M O, Gontijo Filho B, Fantini F A, Martins Jr. I C, Oliveira M H, Avelar SO S, Oliveira O, Vrandecic E, Vrandecic E, Paula e Silva J A - Valva mitral heteróloga sem suporte: resultados clínicos a médio prazo. Rev Bras Cir Cardiovasc 1996; 11 (3): 148-54.

RESUMO: Objetivo:O uso da prótese mitral porcina sem suporte ("Stentless") propicia manutenção das caracteristicas de fluxo e contratilidade do ventriculo esquerdo. No presente estudo, sâo analisados os resultados a médio prazo com o uso desse substituto valvar.

Casuistica e Métodos: No período de março de 1992 a dezembro de 1995, 108 pacientes foram submetidos a implante de valva mitral "Stentless". A idade variou de 11 a 65 anos (média 35,22 $\pm 14,98$ ). A etiologia predominante foi a doença reumática (94 casos), seguida da disfunçāo de bioprótese mitral (6), degeneração mixomatosa (5), endocardite infecciosa (2) e lesăo isquêmica (1). Vinte e seis $(24,1 \%)$ tinham estenose mitral, $24(22,2 \%)$ insuficiência mitral e $58(53,7 \%)$ dupla lesăo. Operaçōes cardiacas prévias haviam sido realizadas em $21,3 \%$ dos pacientes. Procedimentos associados foram necessários em $10(9,3 \%)$ casos.

Resultados:A mortalidade hospitalar foi de 6,5\% (7 pacientes); em apenas 1 caso a endocardite precoce foi relacionada à valva. Dos 101 restantes, 3 foram reoperados, 2 devido a erro na medida da valva e 1 devido a deiscéncia da fixaçăo ao músculo papilar. Com 2 pacientes perdidos no seguimento, 96 foram seguidos por 3,2 a 45 meses. No seguimento tardio ocorreram 6 óbitos devidos a: endocardite (1), infarto agudo do miocárdio (1), pancreatite (1), acidente vascular cerebral (1) e reoperaçס̌es para retroca valvar (2). Foram reoperados tardiamente 12 pacientes, 8 devido a insuficiència mitral ( 1 obito), 2 devido a diminuição da área valvar mitral e 2 a endocardite protética ( 1 obito). Atualmente, 80 pacientes têm sido avaliados trimestralmente. Os estudos ecodopplercardiográficos têm mostrado 63 pacientes com valvas funcionalmente normais, 15 com insuficiência mitral discreta e estável e 2 com reduçăo da área valvar. Com exceçăo destes 2 últimos pacientes, todos os outros têm mostrado melhora da funçāo ventricular esquerda, com reduçāo dos volumes sistólico e diastólico final, em avaliaçōes ecocardiográficas seriadas.

Conclusão: As valvas mitrais porcinas sem suporte têm mostrado melhor performance hemodinămica, com maior possibilidade de manutençäo da funçāo e do tamanho do ventriculo esquerdo. Embora este estudo tenha demonstrado uma curva de aprendizado bem definida relacionada a um novo substituto valvar e à técnica cirúrgica, estes fatores sāo superados com treino e aderência à técnica atualmente em uso.

DESCRITORES: Valva mitral, cirurgia, resultado clínico. Valvas cardiacas, próteses, resultados clínicos. Biopróteses.

Trabalho realizado no Biocór Instituto de Belo Horizonte, MG, Brasil

Apresentado ao $23^{\circ}$ Congresso Nacional de Cirurgia Cardiaca. Recife, PE, 20 a 23 de março, 1996.

"Do Biocór Instituto. Belo Horizonte.

Endereço para correspondẻncia: Mário Vrandecic. Caixa Postal 106. Belo Horizonte, MG, Brasil. CEP:30161-970. Tel. (031) 286-2033.

Fax (031) 286-1688. 
Vrandecic MO, Gontijo Filho B, Fantini F A, Martins Jr. I C, Oliveira M H, Avelar S O S, Oliveira O, Vrandecic E, Vrandecic E, Paula e Silva J A - Valva mitral heteróloga sem suporte: resultados clínicos a médio prazo. Rev Bras Cir Cardiovasc 1996;11 (3): $148-54$

\section{INTRODUÇÃO}

O melhor conhecimento anátomo-funcional da valva mitral, que inclui interaçāo dinâmica do com. plexo mitral e paredes ventriculares, foi um fator determinante da escolha das plastias mitrais, como a técnica ideal no tratamento cirúrgico das valvopatias mitrais $(1-6)$.

A aceitação cada vez maior da importância da preservação do aparelho subvalvar $(7-10)$ na substituição valvar mitral, determinando melhor evoluçäo funcional do ventrículo esquerdo, faz da valva mitral porcina sem suporte ("Stentless Mitral") o substituto natural na troca da valva mitral nos pacientes não candidatos a plastia mitral (11)

A definiçāo estrutural anatômica do complexo mitral ainda é motivo de controvérsia quanto à denominação do número e nome dos elementos anatômicos que a compõem $(10,12-14)$.

A nossa observaçăo é que, para diferenciar dos componentes das valvas aórtica e pulmonar que possuem válvulas das valvas atrioventriculares e, especificamente, na valva mitral, onde reconhecemos "cúspides" aórtica ou anterior, mural ou posterior e duas comissurais.

Este dado anatômico é importante, não só na orientação e implante das cordas tendíneas da "Stentless Mitral", como também na verificação do alinhamento da cordoalha aos trigonos mitro-aórticos.

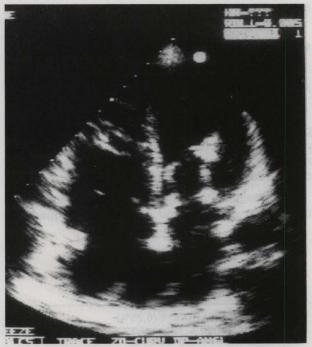

Fig. 1 - Corte apical quatro cámaras mostrando a prótese mitral sem suporte em diástole, com abertura ampla de ambos os folhetos e detalhe do local de sutura do aparelho subvalvar.
TABELA 1

"STENTLESS MITRAL"

N'DE PACIENTES: 108

MARÇO/92 A DEZEMBRO/95
SEXO

Feminino: $67-62 \%$

Masculino: $41-38 \%$

IDADE

Variou de 11 a 65 anos

Média de Idade: $35,22 \pm 14,98$ anos

$<20$ anos: 27 pacientes

O objetivo do presente trabalho é analisar a evolução deste novo substituto valvar mitral quanto aos resultados clínicos, reoperaçōes e características ecocardiográficas.

\section{CASUÍSTICA E MÉTODOS}

No periodo compreendido entre março de 92 a dezembro de 95, 108 pacientes foram submetidos a troca da valva mitral usando o substituto valvar "Stentless Mitral". *

Houve predominância do sexo feminino em 67 $(62 \%)$ pacientes. A idade variou entre 11 e 65 anos (média de 35,22 $\pm 14,98$ anos) (Tabela 1).

A seqüela da doença reumática foi prevalente indicando a troca da valva mitral em 94 pacientes.

Dupla lesão mitral foi predominante em 58 $(53,7 \%)$ pacientes (Tabela 2 ).

Dos 108 pacientes, $85(78,7 \%)$ foram operados pela primeira vez. A condiçāo clínica pré-operatória mostrava 13 pacientes com disfunção ventricular importante, 2 pacientes em sepsis e 1 paciente com acidente vascular cerebral prévio (AVC) secundário a embolia séptica.

TABELA 2

"STENTLESS MITRAL"

NPDE PACIENTES: 108

MARÇO/92 A DEZEMBRO/95

\begin{tabular}{lr}
\hline ETIOLOGıA & \\
Doença Reumática & $94(87,0 \%)$ \\
Disfunçāo da Prótese & $6(5,6 \%)$ \\
Degeneração Mixomatosa & $5(4,6 \%)$ \\
Endocardite Nativa & $2(1,9 \%)$ \\
Isquemia & $1(0,9 \%)$ \\
LESÄO HEMODINĀMICA & \\
Dupla Lesão Mitral & $58(53,7 \%)$ \\
Estenose Mitral & $26(24,1 \%)$ \\
Insuficiència Mitral & $24(22,2 \%)$ \\
\hline
\end{tabular}

- Produzida por Biocór Indústria e Pesquisas Ltda. Belo Horizonte. MG, Brasil. 

e Silva J A - Valva mitral heteróloga sem suporte: resultados clínicos a médio prazo. Rev Bras Cir Cardiovasc1996;11 (3): 148-54.

TABELA 3

"STENTLESS MITRAL"

N DE PACIENTES: 108

MARCCO/92 A DEZEMBRO/95

\begin{tabular}{ll}
\hline \multicolumn{3}{c}{ DIÂMETROS USADOS } \\
25 & $17(15,7 \%)$ \\
27 & $47(43,5 \%)$ \\
29 & $34(31,5 \%)$ \\
31 & $10(9,3 \%)$ \\
\hline
\end{tabular}

Quanto à classe funcional (NYHA), 66 pacientes $(61,1 \%)$ estavam em classe III, $39(36,1 \%)$ em classe IV e apenas 3 pacientes em classe funcional II $(2,8 \%)$.

\section{TÉCNICA CIRÚRGICA}

Embora a técnica cirúrgica seja essencialmente anatômica, a curva de aprendizado é proporcional ao treinamento específico que é indispensável.

Detalhes da técnica cirúrgica foram discutidos préviamente (15-21).

Todos os pacientes foram operados com oxigenador de bolhas, proteçăo miocárdica com cardioplegia cristalóide. O tempo de circulação extracorpórea variou entre 80 a 120 minutos (média de 95 minutos).

O implante da prótese seguiu os principios básicos já com observaçaão da distância intertrigonal para escolha do substituto (os diâmetros mais usados foram 27 e 29), bem como a altura do músculo papilar, anel valvar e correta orientaçăo da "Stentless" na cavidade ventricular (Tabela 3 ).

Procedimentos associados foram realizados em 10 pacientes, sendo a troca da valva aórtica a mais freqüente: $5(4,6 \%)$ pacientes (Tabela 4 ).

\section{RESULTADOS}

A mortalidade hospitalar foi de $7(6,5 \%)$ pacientes $e$ não relacionada ao novo substituto valvar

\section{TABELA 4}

"STENTLESS MITRAL"

$N^{9}$ DE PACIENTES: 108

MARCYO/92 A DEZEMBRO/95

\section{PROCEDIMENTOS ASSOCIADOS}

$\mathrm{N}^{2} 10 / 108$

Troca de Valva Aórtica (biológica)

Comissurotomia Mitral

Encurtamento Cordoalha

Revascularizaçăo Miocárdio

$5(4,6 \%)$

$2(1,9 \%)$

$2(1,9 \%)$

$1(0,9 \%)$
TABELA 5

"STENTLESS MITAAL"

MARCO/92 A DEZEMBRO/95

MORTALIDADE HOSPITALAR: 7 (6,5\%)

$2=$ Acidente Vascular Cerebral

2 = Insuficiência Ventricular

1 = Falência de Múltiplos Órgáos

$1=$ Discrasia Sangûinea

$1=$ Endocardite

COMPLICAÇŌES NĂO FATAIS: $11(10,9 \%)$

4 = Revisăo Hemostática

$4=$ Sindrome de Baixo Débito

$3=$ Reoperaçōes Precoces

em 6 deles. Em 1 paciente 0 óbito foi secundário a endocardite durante o período hospitalar (Tabela 5).

Das complicaçōes não fatais assinaladas, a Tabela 5 mostra que a revisão da hemostasia foi necessária em 4 casos. Três pacientes com insuficiência mitral precoce necessitaram reoperação: em 1 devido a rotura do músculo papilar pósteromedial e em 2 devido a escolha errônea do diâmetro da prótese. Nestes pacientes a "Stentless" foi substituída por bioprótese porcina com suporte. Os 4 pacientes em baixo débito inicialmente responderam bem à terapeutica.

O seguimento pós-operatório foi completo em $98,14 \%$, com exceção de $2(1,86 \%)$ pacientes perdidos durante este seguimento.

O seguimento máximo foi de 45 meses e a média foi de $26,4 \pm 10,1$ meses.

A mortalidade tardia inclui $6(6,25 \%)$ pacientes, sendo as causas indicadas na Tabela 6 .

\section{Reoperaçōes Tardias}

Foram necessárias 12 reoperaçōes tardias em pacientes cujas próteses foram substituidas por bioprótese porcina com suporte. Dois pacientes faleceram: 1 devido a endocardite tardia e outro por rotura das cordas, 15 meses após a operaçāo.

Na maioria das reoperaçōes evidenciou-se má rotaçāo de cordoalha ou tamanho inadequado da

\section{TABELA 6 "STENTLESS MITRAL" MARCYO/92 A DEZEMBRO/95}

MORTALIDADE TARDIA: $6(6,25 \%)$

1 = Infarto Agudo do Miocárdio

$1=$ Acidente Vascular Cerebral

1 = Pancreatite

$1=$ Endocardite

2 = Reoperaçōes para Retroca Valvar 
Vrandecic M O, Gontijo Filho B, Fantini F A, Martins Jr. I C, Oliveira M H, Avelar S O S, Oliveira O, Vrandecic E, Vrandecic E, Paula e Silva J A - Valva mitral heteróloga sem suporte: resultados clínicos a médio prazo. Rev Bras Cir Cardiovasc1996;11 (3): 148-54.

\section{TABELA 7}

"STENTLESS MITRAL" MARCOO/92 A DEZEMBRO/95

REOPERAÇŌES TARDIAS: 12 pacientes $(12,5 \%)$

$B=$ Insuficiência Mitral (1 óbito $)^{*}$

$2=$ Endocardite $(1 \text { b́bito })^{*}$

2 = Estenose Mitral

- Estăo incluídos na mortalidade tardia

"Stentless Mitral" e em 1, deiscência parcial no anel (Tabela 7).

As análises clínica e ecocardiográfica dos pacientes em seguimento $(80)$ mostraram a "Stentless Mitral" competente em $63(78,75 \%)$ pacientes, com regurgitaçāo leve e estável em $15(18,75 \%)$ pacientes e $2(2,5 \%)$ pacientes apresentando diminuiçāo do orificio efetivo da área mitral $(1,3 \mathrm{~cm})$, portanto em risco de reoperação (Tabela 8 ).

Houve melhora evidente da classe funcional no pós-operatório (Gráfico 1).

\section{COMENTÁRIOS}

Toda nova técnica cirúrgica tem sempre uma incidência significativa de reoperaçŏes, embora a experimentação pré-clínica tenha sido adequada.

CARPENTIER (22), pioneiro em plastia mitral, publicou resultados iniciais da técnica no tratamento da insuficiência mitral, mostrando que em 26 de 37 pacientes foi necessária a reoperaçăo, sendo que 18 foram reoperados dentro dos 2 primeiros anos; no ato cirúrgico ficou evidenciado o erro técnico como causa do insucesso em 22 das 26 reoperaçōes.

Das 15 reoperaçōes necessárias neste estudo, 3 foram agudas (nas primeiras 12 horas de evoluçāo) e em retrospecto, previsiveis. As 12 reoperaçōes denominadas tardias ( 3 meses a 3 anos) foram de pacientes operados nos 2 primeiros anos desta experiência.
GRÁFICO 1

"STENTLESS MITAAL" MARCO/92 A DEZEMBRO/95

COMPARAÇĀO DA CLASSE FUNCIONAL PRÉ E POSS-OPERATORIA

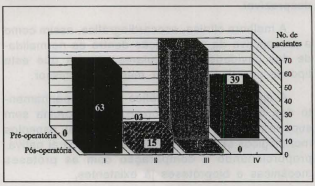

ACAR et al. (23), usando o homoenxerto mitral, salientam a necessidade do domínio da técnica $e$ reconhecem que o uso do anel protético mitral é essencial para diminuir o grau de insuficięncia.

Ao contrário, a "Stentless Mitral" permite o implante, do tamanho ideal para cada paciente (diâmetros existentes variam de $11 \mathrm{~mm}$ a $35 \mathrm{~mm}$ ), evitando-se o uso de prótese cuja evoluçăo tardia associada ao homoenxerto năo é conhecida.

Detalhes, como a determinaçāo correta do diâmetro adequado da prótese, orientaçăo correta da nova origem das cordas no músculo papilar, atençăo ao alinhamento das cordas em relação á via de saida do ventrículo esquerdo, marcada pelo trígono mitro-aórtico direito e esquerdo, assim como mau alinhamento de cordas durante a aproximaçāo dos ânulos é tarefa que requer atenção a cada passo do procedimento e que só é conseguida com o aperfeiçoamento da técnica.

$\mathrm{A}$ anatomia do ventrículo esquerdo e músculos papilares requer os devidos cuidados para que a prótese atenda à individualidade de cada paciente. Este procedimento é, de fato, uma plastia mitral completa.

TABELA 8

"STENTL ESS MITRAL"

MARCO/92 A DEZEMBRO/95

ACHADOS ECOCARDIOGRAFICOS DA "STENTLESS MITRAL"

\begin{tabular}{|c|c|c|c|c|c|c|}
\hline DIĀMETRO & $\begin{array}{l}\text { V. MAX } \\
\text { M/SEG }\end{array}$ & $\begin{array}{l}\text { GRAD. PICO } \\
\text { (MMHG) }\end{array}$ & $\begin{array}{l}\text { GRAD. MÉDIO } \\
\text { (MMHG) }\end{array}$ & $\begin{array}{l}\text { ÁREA VALVAR } \\
\left(\mathrm{CM}_{2}\right)^{*}\end{array}$ & F.E. DO V.E. & $\begin{array}{l}\text { DIMINUIÇĀO DO A.E \% } \\
\text { (MÉDIA) }\end{array}$ \\
\hline 27 & 1,3 & 6,7 & 3,1 & 2,8 & 64 & 26 \\
\hline 29 & 1,3 & 6,7 & 3,0 & 2,8 & 61 & 20 \\
\hline 31 & 1,2 & 5,8 & 2,5 & 2,9 & 63 & 31 \\
\hline \multicolumn{3}{|c|}{$\begin{array}{l}\text { "Pressure Halft Time } \\
\text { Pacientes em repouso }\end{array}$} & \multicolumn{2}{|c|}{$\begin{array}{l}\text { Legenda: } \\
\text { V. Max: Velocidade Máxima } \\
\text { Grad.: Gradiente }\end{array}$} & & $\begin{array}{l}\text { F.E.: Fraçāo de Ejeçāo } \\
\text { V.E.: Ventriculo Esquerdo } \\
\text { A.E.: Atrio Esquerdo }\end{array}$ \\
\hline
\end{tabular}


Vrandecic M O, Gontijo Filho B, Fantini F A, Martins Jr. I C, Oliveira M H. Avelar S O S, Oliveira O, Vrandecic E, Vrandecic E, Paula e Silva J A - Valva mitral heteróloga sem suporte: resultados clínicos a médio prazo. Rev Bras Cir Cardiovasc1996;11 (3): 148-54.

A experiência cirúrgica adquirida nos 2 primeiros anos aprimorou a técnica cirúrgica atual e a incidência de insuficiência valvar importante, hoje desprezivel.

A melhora clínica, ecocardiográfica, assim como a perspectiva da manutenção dentro da normalidade das cavidades ventriculares, mostra que este tipo de substituiçāo valvar mitral é promissor.

A análise ecocardiográfica no acompanhamento dos pacientes com prótese mitral porcina sem suporte mostrou, ao exame bidimensional, aspectos morfológicos semelhantes ao da valva mitral nativa, proporcionando a comparação com as próteses mecânicas e biopróteses já existentes.

A "Stentless Mitral" é superior às próteses cardiacas convencionais usando os parametros descritos na Tabela 8. Observa-se textura normal das cúspides, com excelente mobilidade, abertura e coaptaçăo.

O encurtamento sistólico do "novo ânulo" mitral é evidente, tanto quanto a preservação da função contrátil do ventrículo esquerdo mantendo-se a geometria ventricular.

A análise ao Doppler mostrou suficiência na maioria das próteses; a incidência de regurgitaçāo leve é semelhante à encontrada após as plastias mitrais, e a evoluçăo mostrou estabilidade da mesma.

A área efetiva da prótese mitral estimada ao pressure half time, planimetria ou, pela equação de continuidade, mostrou-se excelente e sempre superior à das próteses convencionais.
Dentro dos seis primeiros meses de seguimento, houve nitida diminuição dos volumes sistólico e diastólico finais no ventrículo esquerdo, com constante melhora dos índices de funçăo sistólica (acima de $30 \%$ ).

Dentro destes 108 pacientes cuja substituição mitral foi realizada com a "Stentless Mitral", 15 pacientes tiveram suas próteses tratadas pelo processo anticalcificante No-React TM e sāo parte de estudo prospectivo de substitutos valvares tratados com a técnica do glutaraldeído convencional e do processo anticalcificante No-React TM.

\section{CONCLUSÃO}

O implante da "Stentless Mitral" provou ser tecnicamente reproduzivel $e$, embora a incidência de reoperaçōes nos dois primeiros anos desta experiência tenha sido significativa, é hoje um procedimento cirúrgico bem estabelecido.

As vantagens hemodinâmicas encontradas foram: reduçāo do diâmetro das cavidades atrioventriculares, gradientes negligenciáveis e melhora da funçāo ventricular na maioria dos pacientes.

Este desenho mitral, aliado ao tratamento anticalcificante No-React TM, mostra ser promissor para alcançar maior sobrevida do paciente e durabilidade do substituto valvar. Todavia, é necessário a confirmação destes bons resultados com estudos clínicos realizados através do tempo. 
Vrandecic MO, Gontijo Filho B, Fantini F A, Martins Jr. I C, Oliveira M H, Avelar S O S, Oliveira O, Vrandecic

E, Vrandecic E, Paula e Silva J A - Heterologous mitral stentless valve: mid-term clinical results. Rev Bras Cir Cardiovasc 1996; 11 (3):148-54.

ABSTRACT: The concept of replacing diseased mitral valve with porcine mitral stentless valve allowed to address the "idiosyncrasy" of the left ventricular flow and contractility. From March 92 to December 95,108 patients had their mitrai valves replaced by stentless mitral valves. Their age varied from 11 to 65 years (mean $35.22 \pm 14.98)$. There were $67(62 \%)$ females and $41(38 \%)$ males. The predominant ethiology was rheumatic heart disease $94(87 \%)$ cases, followed by a prosthetic dysfunction $6(5.6 \%)$ cases, myxomatous disease 5 $(4.6 \%)$ cases, infective endocarditis $2(1.9 \%)$ cases and ischemic lesion $1(0.9 \%)$ case, $26(24.1 \%)$ patients had mitral stenosis, $24(22.2 \%)$ mitral regurgitation and the remaining $58(53.7 \%)$ mixed lesions; $21.3 \%$ of the patients had previous open heart operations. The great majority of the operated patients $(97.2 \%)$ were in functional class III and IV (NYHA). Associated procedures were performed in $9,3 \%(10)$ of the cases.

Results: Hospital mortality occurred in $7(6.5 \%)$ patients non valved related with exception of one whom developed early endocarditis. Of the 101 remaining 3 required reoperations, in two due to valved size mismatch and 1 due to papillary muscle tear. Of the 98 remaining patients, 2 were lost to follow-up, 96 patients have been followed for 3.2 to 45 months. During the late follow-up there were six $(6.25 \%)$ deaths, of the 3 patients with late prosthetic endocarditis, 2 had their valves replaced with standard bioprosthesis, with one death. The third patient expired before reoperation. The late death patient (mitral insufficiency) died early after reoperation. The other 3 patients expired: 1 due to myocardial infarction, 1 due to stroke and 1 with pancreatitis.

Late reoperations: There were 12 patients reoperated, in 8 due to mitral regurgitation with one death, in 2 due to decrease of the mitral valve area, and 2 late endocarditis with one death.

Current Clinical Follow-up: 80 patients are being currently followed. The ecodopplercardiographic studies have shown 63 patients with normal functioning mitral stentless valves, 15 with mild but stable mitral regurgitation and two with reduction on the mitral valve area by the pressure half time. In all but two, serial echocardiographic studies have shown improvement in the left ventricular function, with decreased end systolic and end diastolic volumes.

Conclusion: Porcine mitral stentless valves have shown better performance, are hemodynamicaly superior with greater possibility of maintaining normal ventricular size and function. Although this study showed a distinct "learning curve" related to the new product and technique, these can be overcome by training and following current described operative technique.

DESCRIPTORS: Mitral valve, surgery, clinical results. Heart valves, prostheses, clinical results. Bioprostheses.

\section{REFERÊNCIAS BIBLIOGRÁFICAS}

1 Carabello B A - Preservation of left ventricular function in patients with mitral regurgitation: a realistic goal for the nineties. J Am Cardiol, 1990; 15: 564-5.

2 Gams E, Hagl S, Shcad H, Heimisch W, Mendler N, Sebening F - Importance of the mitral apparatus for left ventricular function: an experimental approach. Eur J Cardiothorac Surg 1983; 6: (Suppl.1): 17-24.

3 Hertz R, Bouriouskas G, Franz M, Borst H G - Mitral valve replacement with preservation of papillary muscles and chordae tendineae: revival of a seemingly forgotten concept. Thorac Cardiovasc Surg 1983; 31: 291-6.

Lillehei C W, Levy M J, Bonnabeau R C - Mitral valve replacement with preservation of papillary muscles and chordae tendinae. J Thorac Cardiovasc Surg $1964 ; 47: 532-43$.

5 Rozich J D, Carabello B A, Usher B W, Krata J M, Bell A E, Ziler M R - Mitral valve replacement with and without chordae preservation in patients with chronic mitral regurgitation. Circulation 1992; 86: 1718-26.

6 Straub $\cup \mathrm{J}$, Huwer $H_{1}$ Petzold $T$, Kalweit $\mathrm{G}$, Volkmer I, Gams E. Preservation of the mitral subvalvular apparatus in multiple valve procedures. $J$ Heart Valve Dis 1995; 4: 477-83.

7 David T E, Armstrong S, Sun Z - Left ventricular function after mitral valve surgery. J Heart Valve Dis 1995; 4 (Suppl.2): S175-80.

8 David T E, Burns R F, Bacchus C M, Druck M N - Mitral valve replacement for mitral regurgitation with and without preservation of the chordae tendinae. $J$ Thorac Cardiovasc Surg 1984; 88: 718-25.

9 David T E \& HO T W - The effect of preservation of chordae tendinae on mitral valve replacement for post-infarction mitral regurgitation. Circulation 1986; 74 (Suppl.1): 116-20.

10 Rusted I E, Scheifley C H, Edwards J E - Estudies of the mitral valve: I. Anatomic features of the normal 
Vrandecic M O, Gontijo Filho B, Fantini F A, Martins Jr. I C, Oliveira M H, Avelar S O S, Oliveira O, Vrandecic E, Vrandecic E, Paula e Silva J A - Valva mitral heteróloga sem suporte: resultados clínicos a médio prazo. Rev Bras Cir Cardiovasc 1996; 11 (3): $148-54$.

mitral valve and associated structures. Br Heart $J$ $1958 ; 6: 825-31$.

11 Yankah A C, Sievers H H, Lange P E, Bernhard A. Clinical report on stentless mitral allografts. J Heart Vaive Dis $1995 ; 4: 40-4$

12 Anderson $\mathrm{RH}$ - Surgical anatomy of the mitral valve (Editorial comments). J Heart Valve Dis 1995; 4: 76-7.

13 Anderson $\mathrm{R} H$ \& Wilcox B R - Understanding cardiac anatomy: the prerequisite for optimal cardiac surgery. Ann Thorac Surg 1995; 59: 1366-75.

14 Kunzeiman K S, Cochran R P, Verrier E D, Eberhart R C. Anatomic basis for mitral valve modelling. $j$ Heart Valve Dis. 1994; 3: 491-6.

15 Vrandecic M, Fantini F A, Gontijo B et al. - Surgical technique of implanting the stentless porcine mitral valve. Ann Thorac Surg 1995; 60: S439-42.

16 Vrandecic M, Gontijo B, Fantini F A et al, - Heterologous mitral valve transplant: the first 50 patients clinical analysis. Eur J Cardio-Thorac Surg 1995; 9; 69-74.

17 Vrandecic M, Gontijo B, Fantini F A et al. - Anatomically complete heterograft mitral valve substitute: surgical technique and immediate results. J Heart Valve Dis 1992; 1: 254-9.
18 Vrandecic M, Gontijo B, Fantini F A et al. - Porcine stentless mitral heart valve substitute: short term clinical data. J Cardiovasc Surg 1994; 35 (Suppl.1 to No.6): $41-5$.

19 Vrandecic M, Gontijo B, Fantini F A et al, - Heterologous stentless mitral valve transplant: a new aproach for mitral valve replacement. Cor Europeaum 1994; 3 : $130-3$

20 Vrandecic M, Gontijo B, Fantini F A et al. - Heterologous mitral valve transplant. New alternative for mitral valve replacement: one year clinical follow-up. In: D'Alessandro L C, ed. Heart surgery 1993. Roma: Casa Editrice Scientifica Internazionale 1993: 57-68.

21 Vrandecic $\mathbf{M}$ O, Gontijo Filho B, Fantini F A et al. Transplante de valva mitral heteróloga. Nova alternativa cirúrgica: estudo clinico inicial. Rev Bras Cir Cardiovasc 1993; 8: 83-90.

22 Carpentier A - Valve reconstruction in predominant mitral valve incompetence. In: Duran C, ed. Recent progress in mitral valve disease. London: Butterworths \& $\mathrm{Co}$. (Publishers) Ltd., 1984; 265-74.

23 Acar C, Farge A, Ramsheyi A et al. - Mitral valve replacement using a cryopreserved mitral homograft. Ann Thorac Surg 1994; 57; 746-8, 